\title{
Магнитные свойства вакансий и внедренного хрома в кристалле ZnO
}

\author{
() В.Н. Джафарова ${ }^{1}$, Г.С. Оруджев ${ }^{1,2}$, С.С. Гусейнова ${ }^{1, \uparrow, ~ В . Р . ~ С т е м п и ц к и и ̆ ~}{ }^{3}$, М.С. Баранава ${ }^{3}$ \\ ${ }^{1}$ Институт фризики Национальной академии наук Азербайджана, \\ Az-1143 Баку, Азербайджан \\ ${ }^{2}$ Азербайджанский технический университет, \\ Az-1143 Баку, Азербайджан \\ ${ }^{3}$ Белорусский государственный университет информатики и радиоэлектроники \\ (НИЛ „Компьютерное проектирование микро- и наноэлектронных систем“), \\ 22013 Минск, Беларусь \\ ฯ E-mail: suma_huseynova.physics@mail.ru \\ (Получена 13 ноября 2017 г. Принята к печати 28 ноября 2017 г.)
}

\begin{abstract}
Представлены результаты теоретических исследований электронных и магнитных свойств $\mathrm{ZnO}$, содержащего дефекты (атом внедрения $\mathrm{Cr}$, вакансии $\mathrm{Zn}$ и O) в кристаллической структуре. Расчеты выполнялись с использованием программных комплексов Atomistix Tool Kit и Vienna Ab-initio Simulation Package, реализующих метод функционала электронной плотности с хаббард коррекцией. Показано, что магнитный момент дефектной суперъячейки имеет сильную зависимость от концентрации примеси и наличия вакансий. Внедрение атома кислорода повышает вероятность образования вакансии цинка.
\end{abstract}

DOI: $10.21883 /$ FTP.2018.08.46219.8678

\section{1. Введение}

Оксид цинка в своей природной модификации имеет гексагональную кристаллическую структуру типа вюрцит [1]. Обладая широкой запрещенной зоной, $\mathrm{ZnO}$ является перспективным прямозонным полупроводником, который при легировании переходными металлами $(\mathrm{Cr}$, $\mathrm{Co}, \mathrm{Fe}, \mathrm{Mn}, \mathrm{Cr}$ или V) проявляет высокотемпературный ферромагнетизм, что было предсказано теоретическими ab initio расчетами, основанными на теории функционала электронной плотности (DFT), и экспериментальными работами [2].

В работе [3], используя опытные измерения и расчеты из первых принципов, изучено влияние вакансий на микроструктуру и ферромагнетизм $\mathrm{ZnO}$ с примесью $\mathrm{Cr}$. В работе [4] представлены результаты анализа возможностей применения оксида цинка, легированного различными переходными металлами, в магнитооптических системах. В работе [5] описаны результаты экспериментальных исследований микроструктуры и магнитных свойств тонких пленок $\mathrm{Zn}_{1-x} \mathrm{Cr}_{x} \mathrm{O}$, осажденных на подложку кварцевого стекла, методом магнетронного радиочастотного напыления.

В настоящей работе представлены результаты квантово-механического моделирования из первых принципов объемного $\mathrm{ZnO}$. В кристаллическую структуру оксида цинка вносились дефекты: внедрение атома $\mathrm{Cr}$, который замещал атом $\mathrm{Zn}$ в узлах кристаллической решетки и собственные вакансионные дефекты.

\section{2. Методы моделирования}

Основные расчеты выполнены с использованием программного пакета Atomistix Tool Kit (ATK), а верификация полученных результатов проведена в программном комплексе Vienna Ab-initio Simulation Package (VASP).
Для описания структурных, электронных и магнитных свойств применялись теория функционала электронной плотности (DFT), аппроксимация локальной спиновой плотности (LSDA) и обменно-корреляционный функционал Perdew-Burke-Erenzhorf (PBE) [6]. Для получения значения ширины запрещенной зоны, близкого к экспериментальному, при моделировании „идеального“ кристалла $\mathrm{ZnO}$ использовалась Хаббард коррекция $(\mathrm{LSDA}+\mathrm{U})$.

Для моделирования влияния примеси переходного металла на спин-зависимые свойства построены суперъячейки, состоящие из 48, 64, 96, 128, 192 атомов. Взаимодействие между атомными остовами и валентными электронами описывалось с помощью псевдопотенциалов Fritz Haber Institute (FHI) и Projector-Augmented Wave (PAW) для ATK и VASP соответственно, а также базисного набора Double Zeta Polarized для программы АТК. В качестве валентных оболочек определены шесть электронов $\mathrm{Cr}\left(3 d^{5} 4 s^{1}\right)$, двенадцать электронов $\mathrm{Zn}\left(3 d^{10} 4 s^{2}\right)$ и шесть электронов кислорода $\left(2 s^{2} 2 p^{4}\right)$.

Исследования выполнялись при следующих условиях: оптимизация геометрии примитивной ячейки $\mathrm{ZnO}$ выполнялась до тех пор, пока силы, действующие на каждый атом, не становились менее чем 0.01 эВ/А, а напряжения $0.01 \mathrm{\ni} / \AA^{3}$. Критерий достижения сходимости расчета при оптимизации суперьячеек, содержащих атомы $\mathrm{Cr}$, считался достигнутым, когда силы, действующие на каждый атом, не превышали значения 0.05 эВ/А.

\section{3. Результаты и обсуждение}

\section{1. Вакансия $\mathrm{Zn}$ и внедрение 0}

На первом этапе по результатам моделирования в программном комплексе АТК магнитных свойств суперъячейки, в составе которой присутствуют собственные 
дефекты, а именно вакансия цинка, установлено, что значение магнитного момента составляет $0.818 \mu_{B}$. Проведя анализ заселенности по Мулликену, определено, что магнитный момент, вносимый 47 атомами цинка, равен $0.099 \mu_{B}$, и $0.719 \mu_{B}$ вносят 48 атомов кислорода. Усиление магнитного момента из-за близости четырех атомов кислорода к вакансии составило $0.384 \mu_{B}$. Участие атомов цинка в намагниченности остается очень слабым $\left(0.099 \mu_{B}\right)$.

Рассмотрен случай, когда в суперьячейку из 96 атомов внедрен атом кислорода. Основным магнитным состоянием системы при этом является ферромагнитное состояние $\left(0.27 \mu_{B}\right.$ вносят 48 атомов $\mathrm{Zn}$ и $1.567 \mu_{B}$ 48 атомов $\mathrm{O}$, полный магнитный момент составил $\left.1.837 \mu_{B}\right)$. Атомы кислорода, находящиеся вблизи вакансии, имеют оборванные связи. По результатам структурной релаксации определено, что два атома кислорода сместились в сторону вакансии, в то время как остальные два атома, напротив, сместились в сторону от вакансии.

Таким образом, внедрение атома кислорода приводит к увеличению полного магнитного момента суперъячейки на $1 \mu_{B}$ по сравнению с его величиной для ячейки, в составе которой присутствует вакансия цинка. Вакансия кислорода совместно с внедренным атомом цинка приводят к антиферромагнитному магнитному упорядочению.

\section{2. $\mathrm{ZnO}$ с внедренным атомом $\mathrm{Cr}$}

Рассчитанная на следующем этапе исследований зонная энергетическая диаграмма показывает, что бездефектный $\mathrm{ZnO}$ представляет собой прямозонный материал с экстремумами, расположенными в центре зоны Бриллюэна. Зонные структуры для подсистем электронов со спинов вверх и со спином вниз имеют одинаковый характер. Ширина запрещенной зоны $(3.4 \ni \mathrm{B})$ и постоянные решетки находятся в хорошем согласии с теоретическими $[3,7]$ и экспериментальными работами

Таблица 1. Структурные параметры ZnO

\begin{tabular}{|c|c|c|c|c|c|c|}
\hline \multirow[t]{2}{*}{ Параметр } & \multirow[t]{2}{*}{ ATK } & \multirow{2}{*}{$\begin{array}{c}\text { Теоретические } \\
\text { значения [3] }\end{array}$} & \multicolumn{4}{|c|}{$\begin{array}{c}\text { Экспериментальные } \\
\text { значения }\end{array}$} \\
\hline & & & {$[3]$} & {$[7]$} & {$[8]$} & [9] \\
\hline$a, \AA$ & 3.2495 & 3.238 & 3.258 & 3.249 & 3.250 & 3.275 \\
\hline$c, \AA$ & 5.2069 & 5.232 & 5.220 & 5.206 & 5.204 & 5.247 \\
\hline$c / a$ & 1.60 & 1.62 & 1.60 & 1.60 & 1.60 & 1.60 \\
\hline
\end{tabular}

Таблица 2. Результаты расчетов магнитных характеристик $\mathrm{Zn}_{1-x} \mathrm{Cr}_{x} \mathrm{O}$

\begin{tabular}{c|c|c|c}
\hline Структура & $c$ & $\mu / \mathrm{Cr},\left(\mu_{B}\right)$ & $\left(E_{\mathrm{AFM}}-E_{\mathrm{FM}}\right) / 2, \mathrm{MэB} / \mathrm{Cr}$ \\
\hline $\mathrm{Zn}_{22} \mathrm{Cr}_{2} \mathrm{O}_{24}$ & $1 / 12$ & 3.97 & 0.825 \\
$\mathrm{Zn}_{46} \mathrm{Cr}_{2} \mathrm{O}_{48}$ & $1 / 24$ & 3.96 & 0.305 \\
$\mathrm{Zn}_{62} \mathrm{Cr}_{2} \mathrm{O}_{64}$ & $1 / 32$ & 3.96 & 248.39 \\
$\mathrm{Zn}_{94} \mathrm{Cr}_{2} \mathrm{O}_{96}$ & $1 / 48$ & 3.96 & 112.505
\end{tabular}

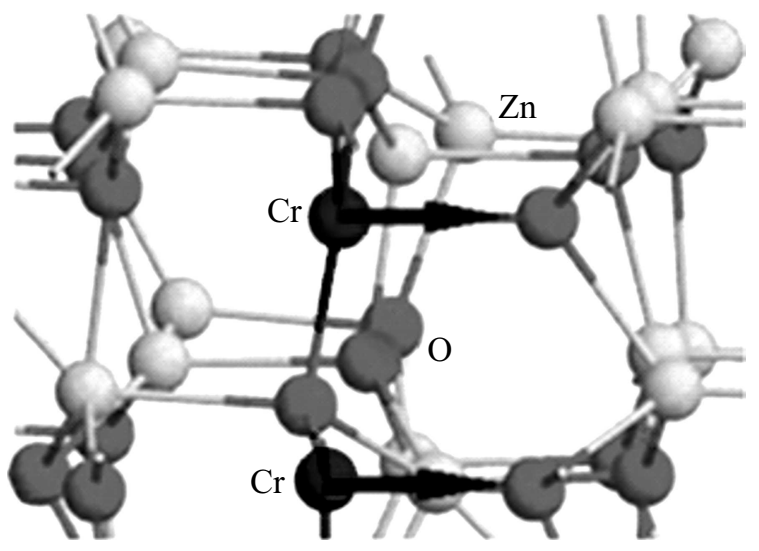

Рис. 1. Расположение дефектов в суперъячейке $\mathrm{Zn}_{46} \mathrm{Cr}_{2} \mathrm{O}_{48}$.

(табл. 1) $[3,8,9]$. Следует отметить, что бездефектный оксид цинка не проявляет ферромагнетизма.

Далее исследована структура $\mathrm{ZnO}$ в которой атом цинка замещен атомом хрома. По результатам моделирования зонных структур и плотности электронных состояний установлено, что значение ширины запрещенной зоны увеличивается до 3.5 эВ.

Возникновение намагниченности в структуре оксида цинка при внедрении атома переходного металла (хрома) объясняется тем, что последний имеет неспаренные по спину электроны. Актуальным является установление по результатам моделирования количественных характеристик магнитных свойств оксида цинка с примесью хрома.

Значения магнитного момента, определенные с помощью анализа заселенности по Мулликену, и разности полной энергии систем с ферромагнитным и антиферромагнитным состоянием приведены в табл. 2.

На основании данных расчетов полной энергии системы установлено, что для исследуемых суперьячеек $\mathrm{Zn}_{x} \mathrm{Cr}_{1-x} \mathrm{O}(x=1 / 12,1 / 16,1 / 24,1 / 32,1 / 48)$ наиболее вероятно ферромагнитное упорядочение. Кристаллическая структура и спиновая поляризация атомов $\mathrm{Cr}$ суперъячейки $\mathrm{Zn}_{46} \mathrm{Cr}_{2} \mathrm{O}_{48}$ представлены на рис. 1 .

На рис. 2 представлены плотности электронных состояний для $s, p$ и $d$ электронов хрома, находящихся в суперъячейке, состоящей из 96 атомов. Верхней кривой соответствуют электроны с направлением спина вверх, а нижней - c направлением спина вниз. Анализ полученных данных свидетельствует о наличии неспаренных электронов преимущественно на $d$ орбитали (рис. 2,c).

\section{3. Примесь $\mathrm{Cr}$ и вакансия $\mathrm{Zn}$}

Исследованы магнитные свойства структуры $\mathrm{ZnO}$, в которой атом цинка замещен атомом хрома, а также присутствует собственный дефект типа вакансии цинка. Результаты моделирования показали, что вклад атома хрома в суммарный магнитный момент составляет $2.173 \mu_{B}$, а вклад четырех атомов кислорода, имеющих 
химическую связь с хромом, равен $-0.143 \mu_{B}$. Вклад остальных 44 атомов кислорода и 46 атомов цинка несуществен $\left(-0.047 \mu_{B}\right.$ и $0.007 \mu_{B}$ соответственно). Полный магнитный момент ячейки $1.989 \mu_{B}$, что меньше на $1.395 \mu_{B}$ по сравнению со структурой, в которой отсутствует вакансия.

Схожие результаты получены для других положений вакансии цинка (рис. 3). Например, если вакансия цинка находится непосредственно возле атома хрома, то

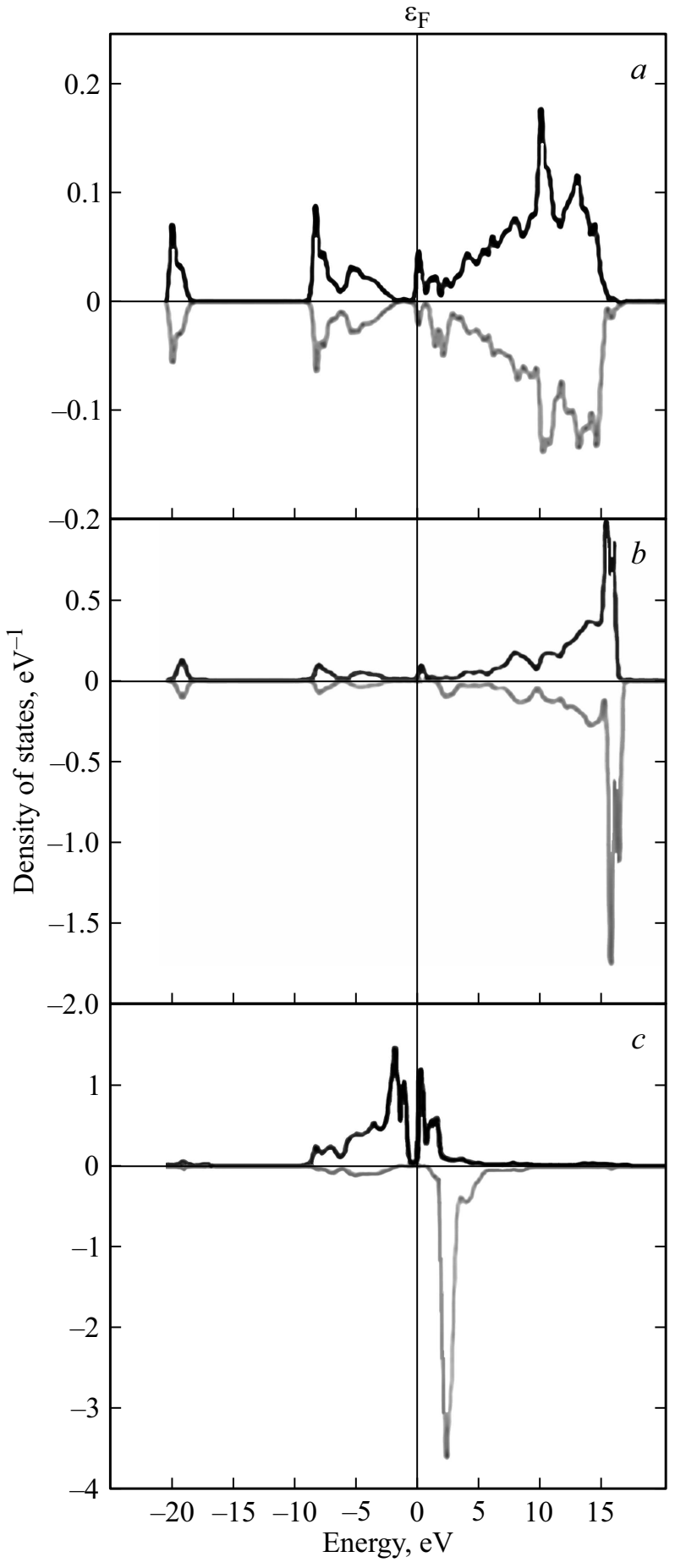

Рис. 2. Плотность электронных состояний для $s, p$ и $d$ электронов атома $\mathrm{Cr}$.

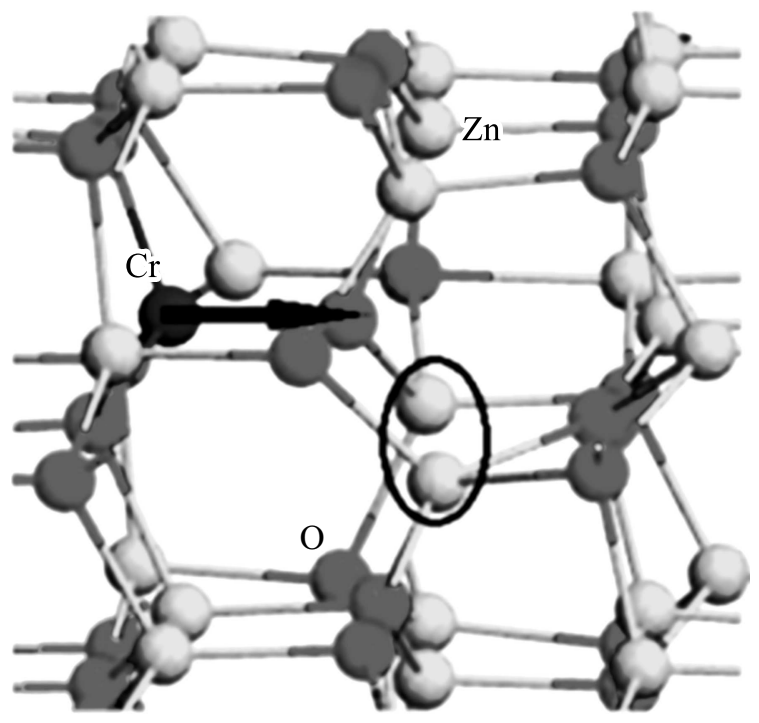

Рис. 3. Расположение дефектов в суперъячейке $\mathrm{Zn}_{47} \mathrm{Cr}_{1} \mathrm{O}_{48}$ с вакансией Zn.

значение полного магнитного момента суперъячейки составляет $1.995 \mu_{B}$ (основной вклад, $2.077 \mu_{B}$ вносит атом $\mathrm{Cr}$, 48 атомов О дают вклад, равный $-0.103 \mu_{B}$, вклад атомов O, находящихся вблизи внедренного атома, составил $-0.094 \mu_{B}$, вклад атомов О, находящихся вблизи вакансии, $\left.0.002 \mu_{B}\right)$.

Таким образом, намагниченность суперъячейки не испытывает сильной зависимости от нахождения вакансии цинка в структуре. Полный магнитный момент суперъячейки при наличии вакансии в структуре, легированной хромом, уменьшается на $1.4 \mu_{B}$. Внедрение атома хрома в суперьячейку $\mathrm{ZnO}$, состоящую из 96 атомов и имеющую вакансию цинка увеличивает магнитный момент на $1.2 \mu_{B}$ по сравнению с системой, в которой находится лишь вакансия.

Вклад в намагниченность четырех атомов кислорода, у которых имеются оборванные связи из-за прямого соседства с вакансией, незначителен. Результаты моделирования структурной релаксации показали, что два атома кислорода будут смещаться в сторону вакансии, в то время как два других атома, напротив, стремятся сместиться в направление от вакансии.

\section{4. Примесь $\mathrm{Cr}$ и вакансия $\mathrm{O}$}

На заключительном этапе исследований проведено $a b$ initio моделирование дефектной структуры $\mathrm{ZnO}$, в которой присутствует вакансия кислорода, расположенная на некотором расстоянии от внедренного атома хрома, замещающего атом цинка в узлах кристаллической решетки. Полный магнитный момент суперъячейки, состоящей из 96 атомов, составил $3.018 \mu_{B}$. Вклад атома хрома составил $3.106 \mu_{B}$, суммарный магнитный момент 47 атомов цинка $0.019 \mu_{B}$ и 47 атомов кислорода 


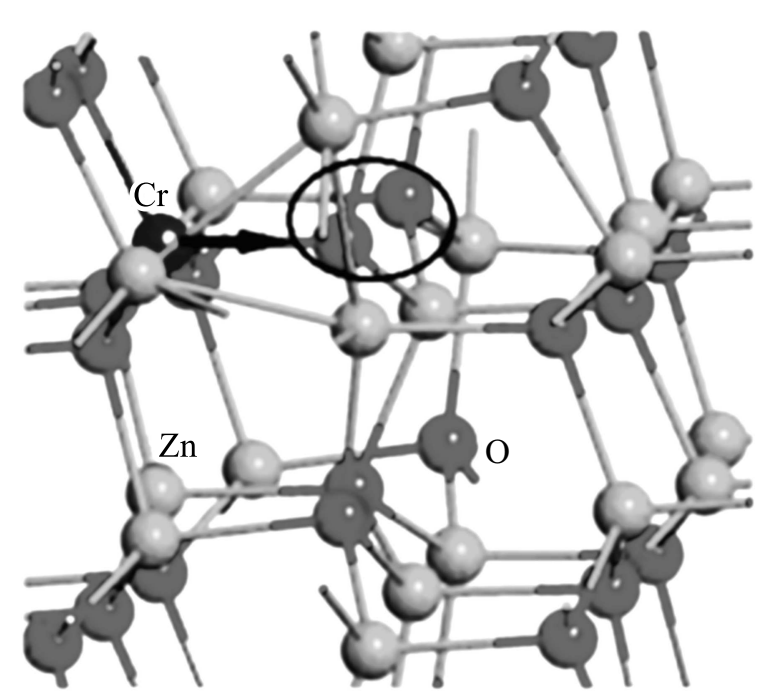

Рис. 4. Расположение дефектов в суперъячейке $\mathrm{Zn}_{23} \mathrm{Cr}_{1} \mathrm{O}_{47}$ с вакансией $\mathrm{O}$.

$-0.107 \mu_{B}$. При отсутствии в подобной системе вакансии и наличии дефекта замещения полный магнитный момент меньше на $0.4 \mu_{B}$.

Для случая, когда дефекты в исследуемой структуре расположены таким образом, что один из атомов цинка имеет химическую связь с примесью хрома, а также оборванную связь из-за наличия вакансии кислорода (рис. 4), полный магнитный момент составил $3.347 \mu_{B}\left(3.131 \mu_{B}\right.$ - вклад атома $\mathrm{Cr}, 0.221 \mu_{B}$ - вклад 47 атомов $\mathrm{Zn}$ и $0.013 \mu_{B}-47$ атомов O). Магнитный момент исследуемой суперьячейки для различного расположения вакансии $\mathrm{O}$ изменялся в диапазоне от 0.037 до $0.366 \mu_{B}$.

Чтобы проверить адекватность полученных ранее результатов, проведено сравнение данных квантовомеханических расчетов для различных конфигураций системы $\mathrm{Zn}_{1-x} \mathrm{Cr}_{x} \mathrm{O}$, выполненных в программном пакете АТК с данными моделирования с использованием программного пакета VASP (табл. 3).

Таблица 3. Сравнение результатов моделирования магнитных характеристик различных конфигураций системы $\mathrm{Zn}_{1-x} \mathrm{Cr}_{x} \mathrm{O}$ в программных пакетах ATK и VASP

\begin{tabular}{c|c|c|c}
\hline Структура & $\begin{array}{c}\mu_{B} \\
(\mathrm{ATK})\end{array}$ & $\begin{array}{c}\mu_{B} \\
(\mathrm{VASP})\end{array}$ & $\begin{array}{c}\left(\mu_{B}(\mathrm{ATK})-\mu_{B}(\mathrm{VASP})\right) / \\
\left(\mu_{B}(\mathrm{ATK})\right), \%\end{array}$ \\
\hline $\mathrm{Zn}_{47} \mathrm{Cr}_{1} \mathrm{O}_{48}$ & 3.3840 & 3.1754 & 6.16 \\
$\mathrm{Zn}_{46} \mathrm{Cr}_{2} \mathrm{O}_{48}$ & 6.7810 & 6.0054 & 11.44 \\
$\mathrm{Zn}_{46} \mathrm{Cr}_{1} \mathrm{O}_{48}$ & 2.0000 & 2.0037 & -0.19 \\
$\mathrm{Zn}_{47} \mathrm{Cr}_{1} \mathrm{O}_{47}$ & 3.3000 & 3.1383 & 4.9 \\
$\mathrm{Zn}_{45} \mathrm{Cr}_{2} \mathrm{O}_{48}$ & 5.9970 & 5.9978 & -0.01 \\
$\mathrm{Zn}_{46} \mathrm{Cr}_{2} \mathrm{O}_{47}$ & 6.6830 & 6.9970 & -4.7 \\
$\mathrm{Zn}_{47} \mathrm{O}_{48}$ & 0.818 & 1.77 & -1.16 \\
$\mathrm{Zn}_{48} \mathrm{O}_{49}$ & 1.837 & 2.00 & -0.089
\end{tabular}

\section{4. Заключение}

Выполнены $a b$ initio расчеты магнитных свойств $\mathrm{ZnO}$. В качестве объектов исследования выбраны суперьячейки монооксида, состоящие из 32, 48, 64, 96, 128, 192 и 256 атомов с различными комбинациями дефектов (внедрение атома хрома и вакансии). Внедрение атома хрома, в качестве атома замещения цинка, приводит к ферромагнитному состоянию всей системы. Установлено, что энергетически более выгодно, а следовательно, вероятнее возникновение дефекта внедрения атома кислорода, чем образование вакансии $\mathrm{Zn}$.

Когда в структуре присутствует вакансия цинка и имплантированный атом кислорода, полупроводник проявляет ферромагнитные свойства, а для комбинаций дефектов, таких как вакансия кислорода и внедренный в междоузлие атом цинка, устанавливается антиферромагнитное состояние. Основные отрицательные вклады в этом случае принадлежат четырем атомам кислорода, которые имеют химическую связь с внедренным хромом. Участие атомов цинка и кислорода в намагниченности пренебрежимо мало. Наличие вакансий в структуре влияет на намагниченность (уменьшает магнитный момент суперъячейки).

Когда атом хрома замещает атом цинка в суперъячейке, состоящей из 96 атомов, значение полного магнитного момента составляет $3.384 \mu_{B}$. Если в подобную структуру ввести дополнительно вакансию цинка или вакансию кислорода, то полный магнитный момент уменьшится до значения $1.395 \mu_{B}$ и $0.366 \mu_{B}$ соответственно.

Работа выполнена при поддержке Фонда развития науки при президенте Азербайджанской Республики (грант № EİF-BGM-2-BRFTF-1-2012/2013-07/03/1-M-05), а также в рамках заданий 2.54 ГПНИ,Физическое материаловедение, новые материалы и технологии“ и 3.02 ГПНИ „Конвергенция-2020“, Государственных программ научных исследований Республики Беларусь.

\section{Список литературы}

[1] T. Makino, Y. Segawa, M. Kawasaki, A. Ohtomo, R. Shiroki, K. Tamura, T. Yasuda, H. Koinumn. Appl. Phys. Lett., 78, 1237 (2001).

[2] K. Sato, H. Katayama-Yoshida. Jpn. J. Appl. Phys., 39, L555 (2000).

[3] C. Jin, Y. Yang, Z.F. Wu, L.Y. Zhuge, Q. Han, X.M. Wu, Y.Y. Li, Z.C. Feng. J. Mater. Chem. C, 2, 2992 (2014).

[4] D. Paul Joseph, C. Venkateswaran. J. Atomic, Molecular, Optical Phys., 2011 (2011).

[5] L. Zhuge, X. Wu, Z.F. Wu, X.M. Chen, Y.D. Meng: Scr. Mater., 60, 214 (2009).

[6] J. Perdew, K. Burke, M. Ernzerhof. Phys. Rev. Lett., 77, 3865 (1996).

[7] A. Arif, O. Belahssen, S. Gareh, S. Benramache. J. Semicond., 36, 013001 (2015).

[8] F. Decremps, F. Datchi, A.M. Saitta, A. Polian, S. Pascarelli, A.Di. Cicco, J.P. Itie, F. Baudelet. Phys. Rev. B, 68, 104101 (2003). 
[9] T. Malaeru, J. Neamtu, C. Moraria, G. Sbarce. Rev. Roum. Chim., 57 (9-10), 857 (2012).

Редактор Г.А. Огагнесян

\section{Magnetic properties of vacancy and $\mathrm{Cr}$-doping states in $\mathrm{ZnO}$}

V.N. Jafarova ${ }^{1}$, G.S. Orudzhev ${ }^{1,2}$, S.S. Huseynova ${ }^{1}$, V.R. Stempitsky ${ }^{3}$, M.S. Baranava ${ }^{3}$

${ }^{1}$ Institute of Physics,

Azerbaijan National Academy of Sciences,

Az-1143 Baku, Azerbaijan

${ }^{2}$ Azerbaijan Technical University,

Az-1073 Baku, Azerbaijan

${ }^{3}$ Belarusian State University

of Informatics and Radioelectronics

(Department of micro- and nanoelectronics),

22013 Minsk, Belarus

Abstract We present a theoretical study of the electronic and magnetic properties of $\mathrm{Cr}$-doped and $\mathrm{Zn}$ - and O-vacanciescontained hexagonal semiconductor $\mathrm{ZnO}$ using density functional calculations. The electronic and magnetic properties of pure as well as Cr-doped $\mathrm{Zn}$ were studied by the DFT $+\mathrm{U}$ method within the local spin density approximation using Atomistix Tool Kit and Vienna Ab-initio Simulation Package programs softwares. The obtained that the magnetic moment of supercell is weakly depending on the dopant concentration and vacancy states. The total energy calculations for a number of supercells showed that in case of implanted oxygen atom is energetically more proferable than zinc-vacancy case. 